\title{
Mapeamento de fontes geradoras de resíduos de serviços de saúde através da utilização de SIG
}

\author{
Mapping of healthcare waste generating sources through the use of GIS
}

\author{
Carolina da Silva Gonçalves ${ }^{1}$ \\ Mateus Torres Nazari ${ }^{2}$ \\ Matheus Francisco da Paz ${ }^{3}$ \\ Diuliana Leandro ${ }^{4}$ \\ Érico Kunde Corrêa ${ }^{5}$ \\ Luciara Bilhalva Corrêa ${ }^{6}$
}

\begin{abstract}
Resumo
Os resíduos de serviços de saúde (RSS) causam impacto à saúde pública e ao meio ambiente quando gerenciados de maneira inadequada. A falta de conhecimento sobre os riscos desses resíduos pelos profissionais da área da saúde e pela população acarretam na destinação incorreta dos mesmos, os quais acabam sendo encaminhados para o programa de coleta seletiva e, posteriormente, às cooperativas de materiais recicláveis. Diante disso, o estudo objetivou analisar as rotas percorridas pelo caminhão da coleta seletiva do Município de Pelotas/RS nas zonas de abrangência do programa e identificar os tipos de fontes geradoras de RSS que se encontram nessas rotas. Por tratar-se de uma pesquisa exploratória, utilizaram-se as ferramentas do Sistema de Informação Geográfica (SIG), que permitem a realização de análises espaciais e geração de informações através de mapas. Foi possível identificar em uma zona 99 estabelecimentos de saúde e, na outra, 15, sendo que esses reúnem tanto atendimento à saúde humana quanto à saúde animal. A presença de RSS em meio a resíduos recicláveis evidencia a necessidade de maiores ações educativas e fiscalizatórias, sendo que, para este último, o SIG pode ser utilizado como uma ferramenta auxiliar.
\end{abstract}

Palavras-chave: Sistemas de informação geográfica. Gerenciamento de resíduos. Saúde pública.

\begin{abstract}
Healthcare waste (HCW) affects public health and the environment when inadequately managed. The lack of knowledge about the risks of such waste by health professionals and by the population leads to the incorrect destination of the waste. It ends up being sent to the selective collection program and, later, to cooperatives of recyclable materials. Thus, this study aimed to analyze the routes traveled by the selective collection truck of
\end{abstract}

\footnotetext{
${ }^{1}$ Universidade Federal de Pelotas, Pelotas, Rio Grande do Sul, Brazil. carolina.engas@gmail.com

2 Universidade de Passo Fundo, Passo Fundo, Rio Grande do Sul, Brazil. nazari.eas@gmail.com

3 Instituto Federal de Educação, Ciência e Tecnologia Sul-riograndense, Pelotas, Rio Grande do Sul, Brazil. matheusfdapaz@hotmail.com

${ }^{4}$ Universidade Federal de Pelotas, Pelotas, Rio Grande do Sul, Brazil. diuliana.leandro@gmail.com

${ }^{5}$ Universidade Federal de Pelotas, Pelotas, Rio Grande do Sul, Brazil. ericokundecorrea@yahoo.com.br

${ }^{6}$ Universidade Federal de Pelotas, Pelotas, Rio Grande do Sul, Brazil. luciarabc@gmail.com
} 
Pelotas/RS in the areas covered by the program to identify the types of HCW generating sources that can be found on these routes. Because this is an exploratory research, we used tools of the Geographic Information System (GIS). It enables the user to perform spatial analysis and data generation through maps. It was possible to identify in one zone 99 health establishments; and, in another, 15 establishments, which refers to both human and animal healthcare. The presence of HWC in recyclable waste evidences the need for greater educational and enforcement actions, and for this last one, GIS can be used as an auxiliary tool.

Keywords: Geographic information system. Waste management. Public health.

\section{Introdução}

Além da geração dos resíduos sólidos domiciliares, que representa um desafio para os gestores públicos e privados, nos ambientes urbanizados são produzidos outros tipos de resíduos, os quais necessitam de gerenciamento adequado (GÜNTHER, 2008). Dentre os resíduos gerados, destacam-se os resíduos de serviços de saúde (RSS), os quais são parte importante do total de resíduos sólidos urbanos, não pela quantidade gerada, cerca de $1 \%$ a $3 \%$ do total, mas pelo potencial de risco que representam à saúde e ao meio ambiente (BRASIL, 2006).

Os riscos inerentes aos RSS devem-se à presença de organismos patogênicos e/ou de toxinas, produtos químicos (fármacos, quimioterápicos, entre outros), além dos riscos radiológicos e perfurocortantes (SCHNEIDER; STEDILE, 2015). Em virtude disso, esses resíduos representam um problema de ordem física, socioeconômica, sanitária e ambiental, desde o seu manuseio, segregação até sua disposição final (TAKAYANAGUI, 2004).

A Lei $\mathrm{n}^{0} 12.305$ de 2010, que institui a Política Nacional dos Resíduos Sólidos (PNRS), responsabiliza todos os geradores pela destinação ambientalmente adequada dos resíduos sólidos. Dentre os objetivos dessa Lei, estão a não geração, a redução, a reutilização, a reciclagem e o tratamento dos resíduos sólidos e a disposição ambientalmente adequada dos rejeitos (BRASIL, 2010). Nesse sentido, uma das diretrizes dessa política é a implantação de programas de coleta seletiva, atividade que representa uma alternativa à reutilização e reciclagem da parte recuperável dos resíduos (CONKE, 2015).

A PNRS ainda estabelece o estímulo e o fortalecimento da coleta seletiva com a integração de catadores organizados em associações ou cooperativas (BRASIL, 2010). De acordo com Cherfem (2016), os catadores procuram pelas associações ou cooperativas em busca de melhores condições de trabalho e segurança social. Contudo, mesmo inseridos nesses locais, esses trabalhadores ainda se encontram expostos a diversos riscos ocupacionais, que segundo Cockell et al. (2004) e Almeida et al. (2009), estão relacionados à incorreta segregação dos resíduos por parte da sociedade, a qual comumente encaminha 
materiais recicláveis misturados a outros tipos de resíduos para a coleta seletiva.

No estudo de Nazari (2017) foram encontrados RSS em cooperativas de catadores na cidade de Pelotas/RS, destinados inadequadamente a estes locais junto aos resíduos recicláveis. Através da caracterização quali-quantitativa de RSS realizada neste estudo foi possível verificar a cooperativa de catadores do município de Pelotas/RS que mais recebia RSS por meio do programa de coleta seletiva, bem como os dias de maior ocorrência desse tipo de resíduo. Com base nisso, foi definido que essa cooperativa seria o foco do presente estudo.

Diante dessa problemática, o objetivo foi analisar as rotas da coleta seletiva do município de Pelotas, Rio Grande do Sul, Brasil, com as coordenadas geográficas latitude $31^{\circ} 46^{\prime} 34^{\prime \prime}$ sul e longitude $52^{\circ} 21^{\prime} 34^{\prime \prime}$ oeste, através da utilização de Sistemas de Informação Geográfica (SIG), a fim de identificar e caracterizar fontes geradoras de RSS.

\section{Metodologia}

A cooperativa estudada foi selecionada com base no estudo de Nazari (2017). A partir dos dias de maior incidência de RSS na cooperativa, sendo eles quinta-feira e sexta-feira, foram delimitados os bairros que encaminhavam resíduos para essa cooperativa. Esses bairros são também conhecidos como "zonas". Essas zonas foram denominadas como Zona 1 e Zona 2 , e posteriormente foram analisadas espacialmente para categorizar as fontes geradoras de RSS e a área de abrangência das rotas percorridas pelo caminhão da coleta seletiva.

Para efetuar a análise espacial e mapeamento das rotas do caminhão da coleta seletiva da cidade, foi utilizado o Programa Quantum Gis (QGIS), versão 2.18.4 (Las Palmas). Este permite a visualização de dados raster e vetoriais, atrelados a um sistema de informação geodésico, proporcionando diversas funções de mapeamento, como: georreferenciamento, análise vetorial e geoprocessamento (TYLLER, 2005; RISTOW, 2014).

Os dados deste estudo (Tabela 1) foram obtidos através das seguintes fontes:

Tabela 1. Base de dados.

\begin{tabular}{cc}
\hline Dados & Fonte \\
Mapa de Pelotas & Site Infraestrutura Nacional de Dados Espaciais (INDE) \\
Quadras de Pelotas & Mapa Urbano Básico (MUB) da Prefeitura de Pelotas cedido pela Defesa \\
Civil & MUB da Prefeitura de Pelotas cedido pela Defesa Civil \\
Letes das Quadras & Plano Municipal de Gestão Integrada de Resíduos Sólidos (PMGIRS, 2014) \\
de Pelotas & Site Google Maps \\
\hline
\end{tabular}

Org.: da Autora, 2017. 
Foi realizado o delineamento da rota percorrida pelo caminhão da coleta seletiva. Esse procedimento delimitou-se as zonas que destinam os resíduos para a cooperativa analisada. Em seguida, realizou-se a vetorização da zona, utilizando como norteador o mapa da coleta seletiva do município e as plantas de roteiro da coleta de resíduos, contidos no PMGIRS (2014).

As fontes geradoras de RSS foram identificadas através do programa Google Maps, adotando a metodologia de Silva (2004), que atribui a cada ponto a sua localização espacial (coordenadas X,Y). Para a categorização dessas fontes foi empregada a ferramenta "Street View" do Google Maps, com a função "Satélite" acionada. Logo, percorreu-se o trajeto do caminhão da coleta seletiva e identificou-se a fonte geradora pela sua fachada. As coordenadas geográficas das fontes foram exportadas para o Programa QGIS e adicionadas ao lote pertencente.

Diante disso, buscou-se a exploração da área de abrangência da rota, visto que essa também pode englobar outras fontes que aproveitam essa rota para destinar resíduos às cooperativas. Para a realização dessa análise espacial, tomou-se como base a rota vetorizada.

Para a etapa de confecção dos mapas foram utilizados os dados: tipos de fontes geradoras, rota e área de abrangência. A partir disso, foi definido o conjunto de cores adequados através do software ColorBrewer 2.0 (BREWER, 2009), o qual oferece uma série de esquemas de cores para mapas temáticos que permite a representação e mapeamento de dados de forma clara (LEANDRO, 2013).

O Sistema de Referência e o de Projeção utilizados foram o Sistema de Referência Geocêntrico para as Américas 2000 (SIRGAS 2000) e o Universal Transversa de Mercator (UTM) zona $22 \mathrm{Sul}$, respectivamente. $\mathrm{O}$ código de Sistemas de Referência de Coordenadas encontrado no programa foi o European Petroleum Survey Group (EPSG): 31982, na escala 1:2000.

\section{Resultados e Discussão}

O Mapa 1 mostra as fontes geradoras de RSS encontradas na Zona 1. Nota-se a presença significativa de 99 geradores, dos quais a maioria se encontra próxima a rota percorrida pelos caminhões. As fontes geradoras de RSS apontados nessa zona localizam-se em grande parte próximos as vias de acesso frequente da população. Com isso, deve-se atentar ao descarte de resíduos sólidos desses locais, uma vez que na coleta seletiva porta a porta os recicláveis encontram-se em frente as residências e estabelecimentos. 
Mapa 1 - Fontes geradoras de RSS encontradas na Zona 1

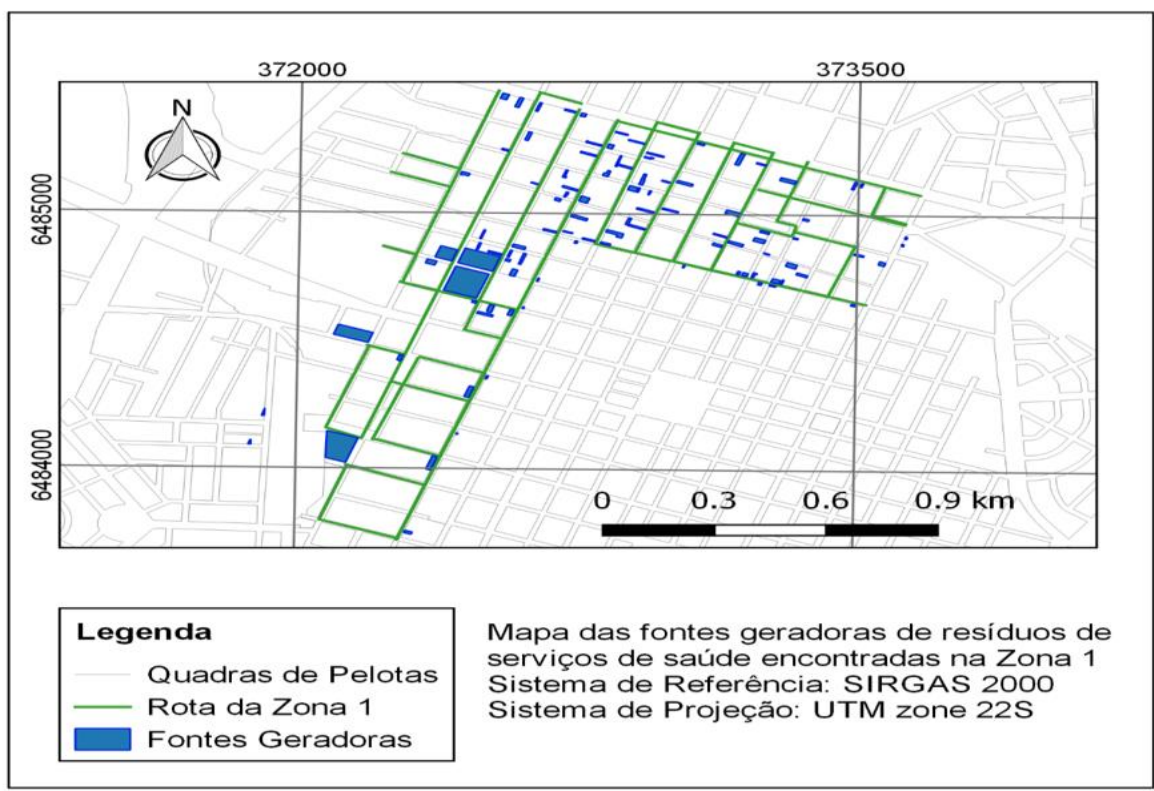

Fonte: Serviço Autônomo de Saneamento de Pelotas (SANEP), 2014; Google Maps, 2017. Org.: da Autora, 2017.

Richter (2014) enfatiza que os resíduos depositados em calçadas para o recolhimento pela coleta seletiva podem causar alguns problemas em relação à animais, que por muitas vezes rasgam as sacolas procurando por alimentos, enquanto que o vento e a chuva acabam por carregar os resíduos contidos nas sacolas, propiciando a proliferação de mau cheiro e de vetores que podem causar doenças. Além disso, o autor menciona a situação dos catadores autônomos que coletam os materiais recicláveis, segregando-os dos demais resíduos e, muitas vezes, espalhando-os pela via pública, estando propícios a contrair doenças.

\section{Nesse contexto de inadequada} segregação, salienta-se que os RSS descartados juntos aos recicláveis destinados à coleta seletiva podem acarretar em diversos riscos à saúde da comunidade, uma vez que diferentes atores da cadeia de resíduos entram em contato, direta ou indiretamente, com materiais infectantes ou contaminantes, causando situações de prejuízo ambiental que ultrapassam os limites dos locais de geração (SCHNEIDER et al., 2015).

Uma correta segregação proporciona uma diminuição da quantidade de RSS infectados, impossibilitando a contaminação da massa total dos resíduos gerados, o que impede que ocorram fatos tipificados como crimes ambientais (TAKADA, 2003). O autor enfatiza que a ausência ou inadequada segregação dos resíduos biológicos propicia o contato entre esses e os resíduos comuns, tornando-os também infectantes, aumentando o risco para os trabalhadores da saúde e a população em geral.

O Mapa 2 apresenta a Zona 2 que também foi apontada como uma das que contribuiu para o encaminhamento inadequado 
de RSS à cooperativa. Nessa Zona foi possível verificar a presença de 15 geradores. Em comparação à Zona 1, a quantidade encontrada é expressivamente inferior. Um dos fatores justificativos para esse fato pode ser que no processo de identificação, nem todos os estabelecimentos foram apontados devido à dificuldade de visualização das fachadas dos mesmos e, também deve-se considerar que existam locais sem identificação. Além disso, nota-se que as fontes geradores de RSS encontradas estão próximas a rota em que o caminhão percorre durante o recolhimento dos resíduos.

Mapa 2 - Fontes geradoras de RSS encontradas na Zona 2.

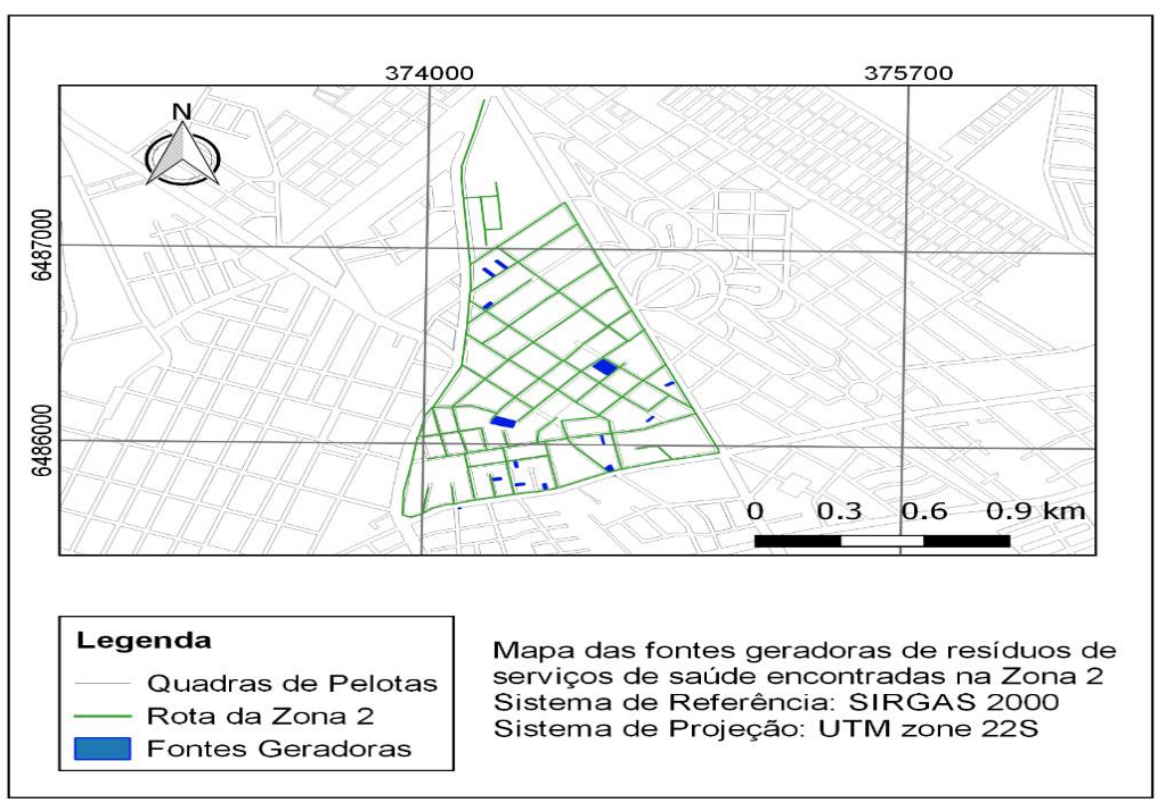

Fonte: SANEP, 2014; Google Maps, 2017.

Org.: da Autora, 2017.

Para os tipos de fontes geradoras foi utilizada foi realizada a separação em 10 categorias para uma melhor qualificação e quantificação, sendo elas: clínica, consultório, equipamentos hospitalares, estúdios de tatuagem, farmácias, funerárias, hospitais, laboratórios de análises clínicas, veterinárias/pet shops e outros.

É importante destacar que os RSS não se restringem apenas aos resíduos gerados nos hospitais, mas também a todos os demais estabelecimentos geradores de resíduos de saúde, tais como: laboratórios patológicos e de análises clínicas, clínicas veterinárias, centros de pesquisas, laboratórios, banco de sangue, consultórios médicos, odontológicos e similares (BRASIL, 2005).

A relação das categorias de fontes geradoras de RSS encontradas na Zona 1 pode ser observada no Mapa 3. 
Mapa 3 - Tipos de fontes geradoras de RSS encontrados na Zona 1.

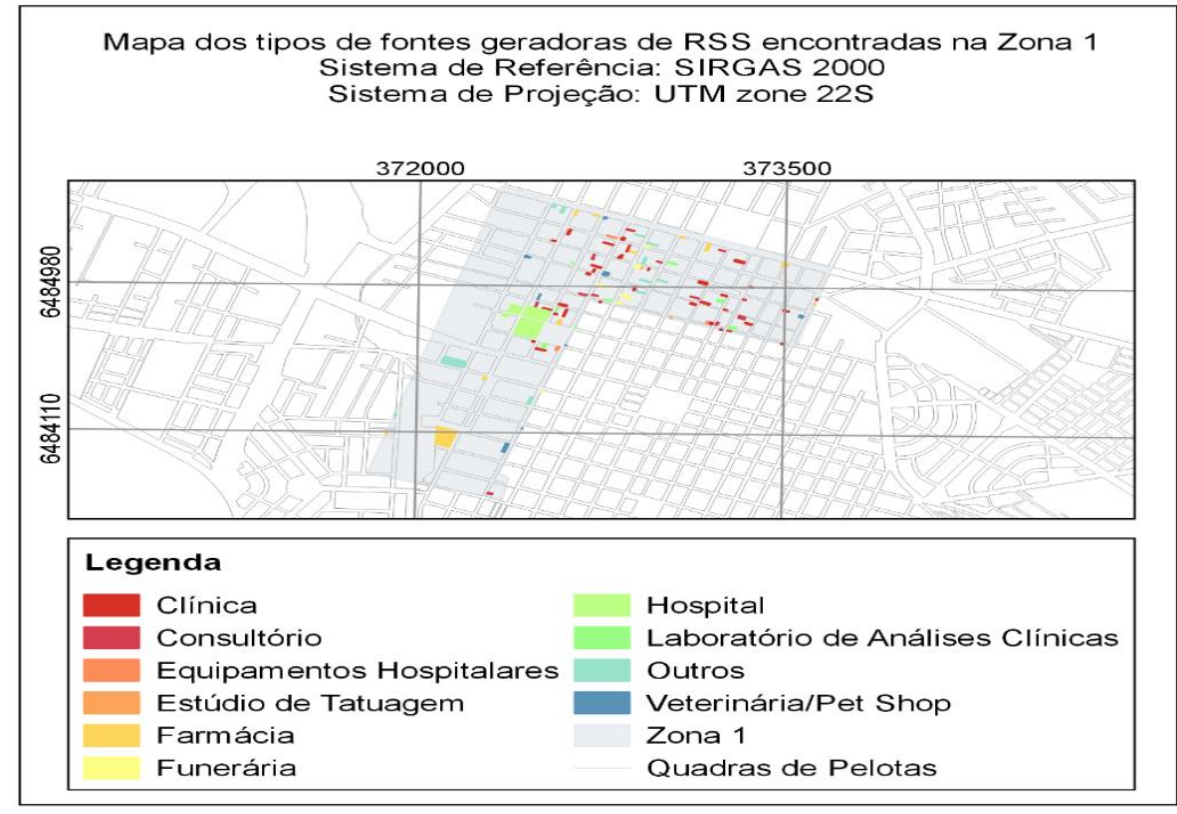

Fonte: Google Maps, 2017.

Org.: da Autora, 2017.

Foram encontradas 33 clínicas, caracterizadas em: odontológicas, estéticas, ginecológicas, pediátricas, especialidades, além das de atendimento geral. Em relação aos nove laboratórios de análises clínicas encontrados, englobam-se os de análises humanas e os de análises veterinárias. Já nos equipamentos hospitalares, foram quatro, entre os quais estão inseridos os prestadores de serviços desses equipamentos aos estabelecimentos de saúde.

Foram encontrados 11 consultórios, especificados em: odontológicos, estéticas, pediátricos e clínico geral. Foram localizados ainda seis funerárias, 12 farmácias e um estúdio de tatuagem.

Foram identificados oito estabelecimentos de veterinária e pet shop, que foram relacionados em apenas um grupo para melhor visualização. Destaca-se que muitos pet shops possuem serviços de assistência à saúde animal. $\mathrm{Na}$ categoria dos hospitais, foram visualizados quatro, englobando um ambulatório, que foi atrelado a esse tipo de gerador. Por fim, a categoria "outros" contempla 11 estabelecimentos, entre os quais estão os lares para idosos, lares para crianças, casas geriátricas, casas de amparo social e a Secretaria Municipal de Saúde.

O Mapa 4 mostra as fontes geradoras de RSS na Zona 2. As cinco clínicas identificadas se dividem em estéticas, geriátricas, dentárias e clínica de atendimento geral. A mesma quantidade de clínicas foi encontrada na categoria veterinária e pet shop. $\mathrm{O}$ único consultório era de caráter dentário, enquanto o hospital foi caracterizado como uma Unidade Básica de Saúde. Além disso, foi localizada uma farmácia nessa rota da coleta seletiva. 
Mapa 4 - Tipos de fontes geradoras de RSS encontrados na Zona 2.

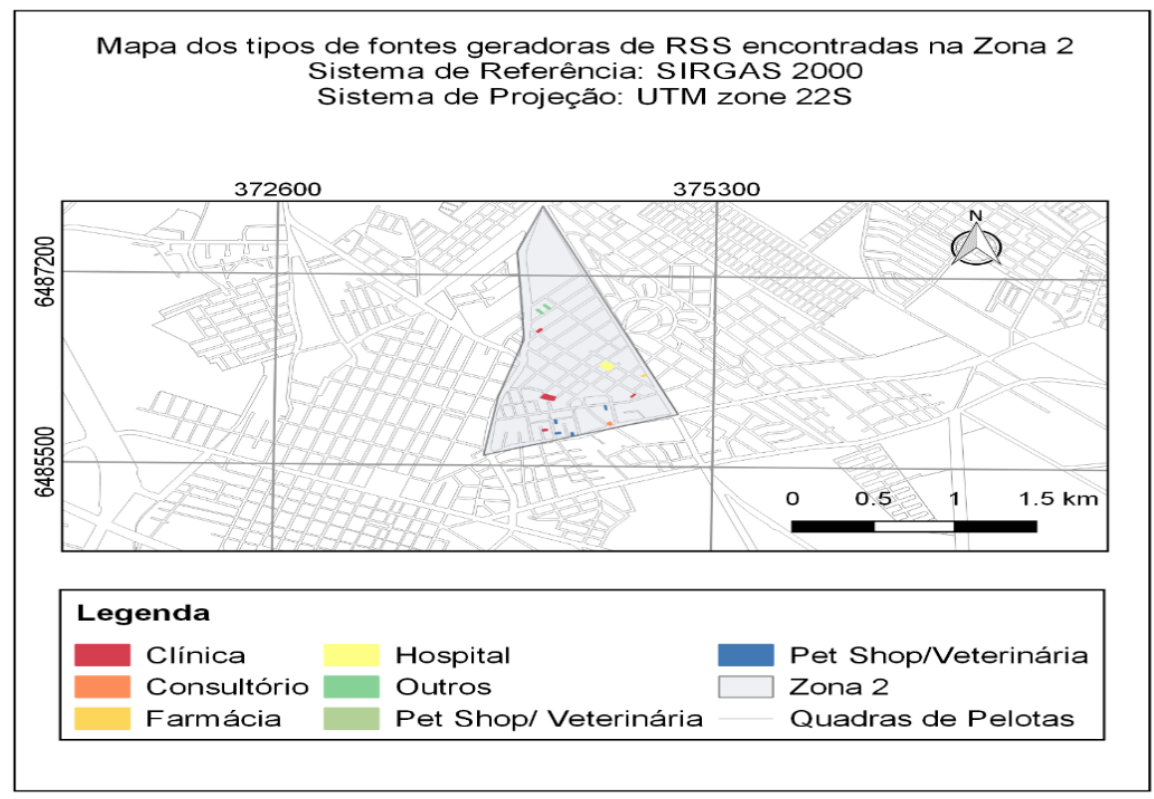

Fonte: Google Maps, 2017.

Org.: da Autora, 2017.

No que diz respeito à abrangência das rotas, pode-se verificar no Mapa 5 e no Mapa 6 o delineamento dessas e o quanto elas podem atingir em uma distância de 100 metros.

Mapa 5 - Distância de 100 metros da rota do caminhão da coleta seletiva que passa pela Zona 1.

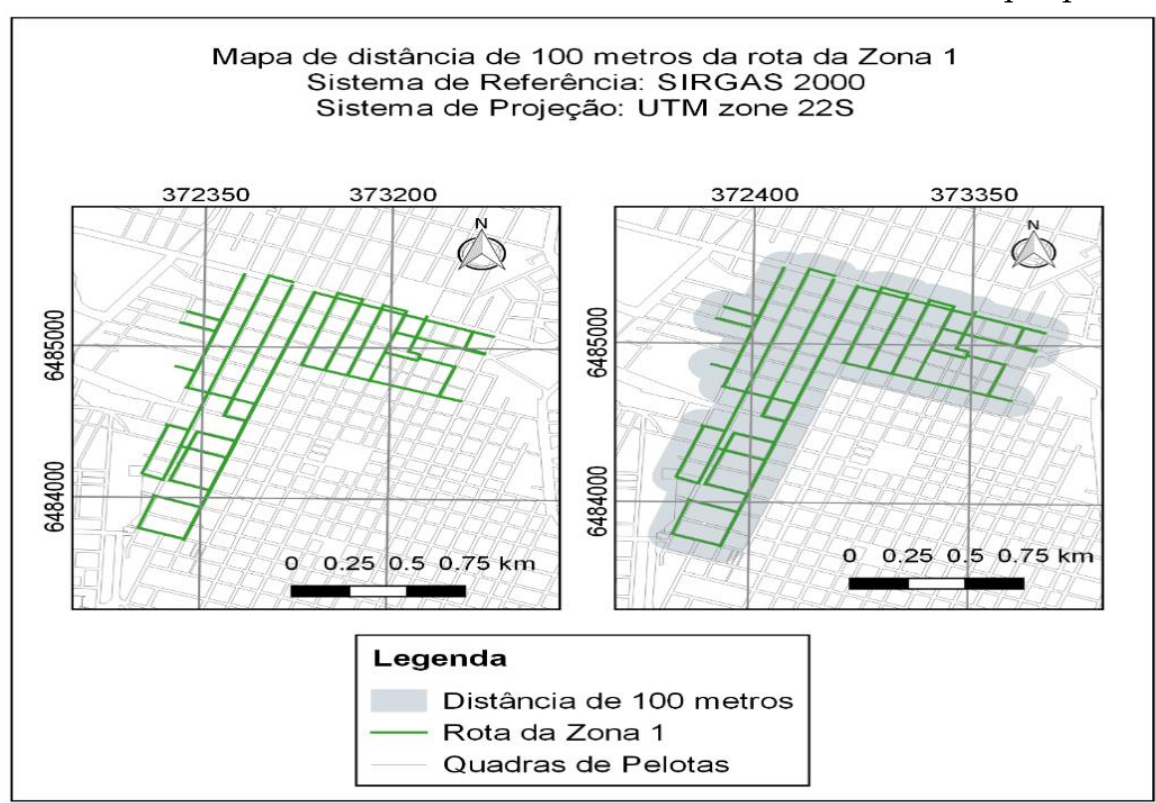

Fonte: SANEP, 2014.

Org.: da Autora, 2017. 
Mapa 6 - Distância de 100 metros da rota do caminhão da coleta eletiva que passa pela Zona 2.

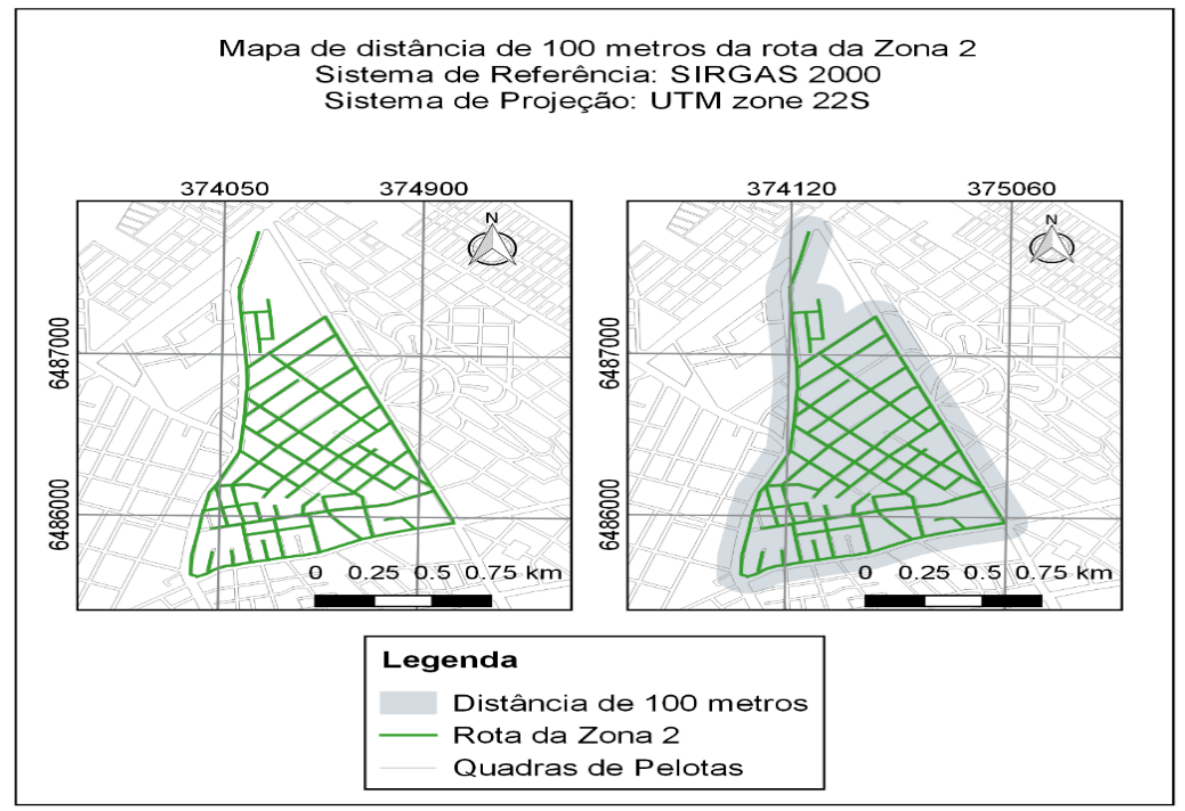

Fonte: SANEP, 2014.

Org.: da Autora, 2017.

Diante dessas figuras e da análise espacial, nota-se que a referente distância de 100 metros engloba outras quadras que ultrapassam as delimitações das zonas. As análises mostraram que além dos estabelecimentos de saúde encontrados nas rotas, outros geradores não incluídos diretamente na rota da coleta seletiva, também podem ser possíveis destinadores de RSS às cooperativas.

A aparente irresponsabilidade dos geradores em não realizarem uma adequada segregação desses resíduos, bem como a falta de conscientização e desinformação dos riscos à saúde pública ao enviar esses tipos de resíduos sólidos junto aos resíduos recicláveis para o programa de coleta seletiva caracterizam uma grave falha na gestão de resíduos.
Para Corrêa et al. (2007) a segregação de RSS relaciona-se a ética dos profissionais que atuam nos estabelecimentos de saúde, uma vez que o gerenciamento desses resíduos nas fontes geradoras depende, principalmente da colaboração dos atuantes com o correto manejo. Os autores destacam que essa consciência dos profissionais reflete na responsabilidade com o meio ambiente e na melhoria da qualidade de vida do estabelecimento, dos pacientes, da comunidade em geral e de todos os profissionais que lidam diretamente na cadeia dos resíduos.

Além disso, percebe-se a carência de fiscalização dos órgãos públicos licenciados na verificação da conduta do gerenciamento dos RSS e da elaboração e implementação de Plano de Gerenciamento de Resíduos de Serviços de Saúde (PGRSS) em estabelecimentos de 
atendimento à saúde. Esse plano objetiva promover o bem-estar do profissional de saúde no seu ambiente de trabalho, tal como da comunidade em geral (TAKADA, 2003).

Com relação ao SIG, da mesma forma que se utilizou essa ferramenta para este estudo, Máximo (2004) empregou essa tecnologia para realizar o mapeamento da criminalidade urbana e verificaram sua utilidade na segurança pública. Ainda, Carvalho et al. (2007) reportam para a abordagem espacial com o uso de SIG na área da saúde, realizando a análise espacial associando eventos de saúde com a utilização de indicadores e/ou variáveis socioambientais, sendo úteis no Sistema de Vigilância em Saúde.

\section{Considerações Finais}

O emprego da análise espacial através de SIG no gerenciamento de resíduos é uma temática a ser explorada, principalmente por sua versatilidade e maior utilização nos últimos anos. Através do mapeamento realizado neste estudo foi possível delimitar as zonas de abrangência da coleta seletiva, além de quantificar e qualificar as fontes geradoras de RSS, classificando-os em categorias.

Foram identificados

estabelecimentos de saúde de atendimento à saúde humana e animal em duas zonas pertencentes ao programa da coleta seletiva. Também foi possível classificar diferentes tipos de fontes geradoras de RSS, sendo a categoria "clínicas" a mais encontrada, com 33 e 5 estabelecimentos, na Zona 1 e na Zona 2, respectivamente.

Por fim, a correlação entre a incidência de RSS na cooperativa de material reciclável com o mapeamento das fontes geradoras de RSS através do Programa QGIS pode facilitar os serviços de fiscalização e, com isso, zelar pela saúde ambiental e, principalmente, dos profissionais envolvidos diretamente com o gerenciamento de resíduos.

\section{Referências}

ALMEIDA, J. R.; ELIAS, E. T.; MAGALHÃES, M. A.; VIEIRA, A. J. D. Efeito da idade sobre a qualidade de vida e saúde dos catadores de materiais recicláveis de uma associação em Governador Valadares, Minas Gerais, Brasil. Ciência \& Saúde Coletiva, v. 14, n. 6 , p. 2169-2180, 2009 . http://dx.doi.org/10.1590/S141381232009000600024

BRASIL. Resolução n. ${ }^{\circ} \mathbf{3 5 8}$, de 29 de abril de 2005. Disponível em: <http://www2. mma. gov. br/port/conama/legiabre.cfm?codlegi $=462>$. Acesso em: 03 de dezembro de 2019

Ministério da Saúde. Agência Nacional de Vigilância Sanitária (ANVISA). Manual de Gerenciamento dos Resíduos de Serviços de Saúde: Tecnologia em Serviços de Saúde. 1. ed, Brasília: ANVISA, 2006.

Lei n. $^{\circ} 12.305$, de 2 de agosto de 2010. Disponível: <http://www.planalto.gov. br/ccivil_03/ ato20072010/2010/lei/112305.htm>. Acesso em: 03 de dezembro de 2019.

BREWER, C. A. ColorBrewer 2. 0. Software, Geography, Pennsylvania State University. $2009 . \quad$ Disponível em: $<$ http://colorbrewer2.org/\#type=sequential\&sc heme=BuGn\&n=3>. Acesso em: 08 de julho de 2017.

CARVALHO, M. S.; CRUZ, O. G.; SOUZA, W. V.; MONTEIRO, A. M. V. Conceitos básicos em análise de dados espaciais em saúde. In: SANTOS, S. M.; SOUZA, W. V. (Orgs.) Introdução à Estatística Espacial para a Saúde Pública. Brasília: Ministério da Saúde, 2007. p 13-27. 
CHERFEM, C. O. Relações de gênero e raça em uma cooperativa de resíduos sólidos: desafios de um setor. In: PEREIRA, B. C. J.; GOES, F. L. (Orgs.) Catadores de materiais recicláveis: em encontro nacional. Rio de Janeiro: IPEA, 2016. p. 47-74.

COCKELL, F. F.; CARVALHO, A. M. C. de; CAMAROTTO, J. A.; BENTO, P. E. G. A triagem de lixo reciclável: análise ergonômica da atividade. Revista Brasileira de Saúde Ocupacional, v. 29, n. 110, p. 17-26, 2004. http://dx.doi.org/10.1590/S030376572004000200003

CONKE, L. S. Barreiras ao Desenvolvimento da Coleta Seletiva no Brasil. Tese (Doutorado em Desenvolvimento Sustentável) - Brasília: UnB. 2015.

CORREAA, L. B.; LUNARDI, V. L.; DE CONTO, S. M. O processo de formação em saúde: o saber resíduos sólidos de serviços de saúde em vivências práticas. Revista Brasileira de Enfermagem, v. 60, n. 1, p. 21-25, 2007. http://dx.doi.org/10.1590/S003471672007000100004

GÜNTHER, W. M. R. Resíduos sólidos no contexto da saúde ambiental. Tese (Livre Docência) - São Paulo: USP. 2008.

LEANDRO, D. Modelagem de fragilidade ambiental usando índices baseados em dados especiais e com suporte de sistema especialista. Tese (Doutorado em Ciências Geodésicas) - Curitiba: UFPR. 2013.

MÁXIMO, A. A. A importância do mapeamento da criminalidade utilizando-se tecnologia de sistema de informação geográfica para auxiliar a segurança pública no combate à violência. 2004. $101 \mathrm{f}$. Dissertação (Mestrado em Engenharia de Produção) - Universidade Federal de Santa Catarina, Florianópolis, 2004.

NAZARI, M. T. Incidência de resíduos de serviços de saúde nas cooperativas de triagem de materiais recicláveis do Município de Pelotas/RS. 2017. 59 f. Trabalho de Conclusão de Curso (Bacharelado em Engenharia Ambiental e Sanitária) Universidade Federal de Pelotas, Pelotas, $2017 . \quad$ https://doi.org/10.5151/engproeneeamb2016-rs-002-4936
PMGIRS - Plano Municipal de Gestão Integrada de Resíduos Sólidos - Pelotas. $2014 . \quad$ Disponível em: $<$ https://docplayer.com.br/9894849-Planomunicipal-de-gestao-integrada-de-residuossolidos-pmgirs-municipio-de-pelotas-rs.html >. Acesso em: 28 de julho de 2017.

RICHTER, L. T. A importância da conscientização e da coleta seletiva no município de Palmitos-SC. 2014. 78 f. Monografia (Especialização em Gestão Ambiental em Municípios) - Universidade Tecnológica Federal do Paraná, Medianeira, 2014.

RISTOW, S. F. P (Org.). Uso de geotecnologias livres para apoio à gestão de bacias hidrográficas: prática com Quantum Gisversão 2. 2. 0. Florianópolis: UFSC, 2014.

SCHNEIDER, V. E.; STEDILE, N. L. R.; BERGER FILHO, A. G. Potencial de risco dos resíduos de serviços de saúde. In: SCHNEIDER, V. E.; STEDILE, N. L. R (Orgs.). Resíduos de Serviços de Saúde: um olhar interdisciplinar sobre o fenômeno. Caxias do Sul: Educs, 2015. p. 57-78.

SCHNEIDER, V. E.; STEDILE, N. L. R (Orgs.). Resíduos de Serviços de Saúde: um olhar interdisciplinar sobre o fenômeno. Caxias do Sul: Educs. 3. ed., ampl. e atual, 2015.

SILVA, S. A. Utilização de técnicas de análise espacial como ferramenta para vigilância de pneumonias radiologicamente definidas na infância em Goiânia. Tese (Doutorado - Área de Concentração de Epidemiologia) - Goiânia: UFG. 2004.

TAKADA, A. C. da S. O plano de gerenciamento dos resíduos de serviços de saúde e o direito do trabalhador. 2003. 35 f. Monografia (Especialização em Direito Sanitário para Profissionais da Saúde) Escola Nacional de Saúde Pública Sérgio Arouca, Brasília, 2003.

TAKAYANAGUI, A. M. M. Risco ambiental e o gerenciamento de resíduos nos espaços de um serviço de saúde no Canadá: um estudo de caso. Tese (Livre Docência) Ribeirão Preto: EERP USP. 2004.

TYLLER, M. Introdução a SIG. In: CENSIPAM (Org.). Manual do usuário Quantum Gis versão 1.4.1 "Enceladus". Brasília: CENSIPAM, 2005. p. 11-13. 